\title{
Finite Volume Effects in Weak Hadronic Decays *
}

\author{
C.-J.D. Lin ${ }^{\mathrm{a}}$, G. Martinelli ${ }^{\mathrm{b}}$, C.T. Sachrajda ${ }^{\mathrm{a}}, \mathrm{M}$. Testa $^{\mathrm{b}}$. \\ ${ }^{a}$ Dept. of Physics and Astronomy, Univ. of Southampton, \\ Southampton, SO17 1BJ, UK \\ ${ }^{\text {b}}$ Dip. di Fisica, Univ. di Roma "La Sapienza" and INFN, Sezione di Roma, \\ Piazzale Aldo Moro 2, I-00185 Rome, Italy
}

\begin{abstract}
In this talk we discuss finite-volume computations of two-body hadronic decays below the inelastic threshold (e.g. $K \rightarrow \pi \pi$ decays). In particular we show how the relation between finite-volume matrix elements and physical amplitudes, recently derived by Lellouch and Lüscher, can be extended to all elastic states under the inelastic threshold. We also provide a derivation of the Lüscher quantization condition directly in quantum field theory.
\end{abstract}

\section{Introduction}

Lattice QCD offers a natural opportunity to compute the matrix elements for $K \rightarrow \pi \pi$ decays from first principles. The main difficulties are related to the continuum limit of the regularized theory (the ultra-violet problem) and to the relation between matrix elements computed in a finite Euclidean space-time volume and the corresponding physical amplitudes (the infrared problem). The ultra-violet problem, which deals with the construction of finite matrix elements of renormalized operators from the bare lattice ones, has been addressed in a series of papers [1]-[3] and we will not consider it further. The infrared problem arises from two sources:

- the unavoidable continuation of the theory to Euclidean space-time and

- the use of a finite volume in numerical simulations.

One of the main obstacles in the extraction of physical amplitudes from lattice simulations stems from the rescattering of final state particles in Euclidean space. The formalization of this problem, in the infinite-volume case, was considered in ref. [4]. An important step towards the solution of the infrared problem has recently been

\footnotetext{
*Talk presented by M. Testa at the Workshop on Lattice
} Hadron Physics (LHP2001), Cairns, Australia achieved by Lellouch and Lüscher [5] (LL), who derived a relation, given below in eq.(11), between the $K \rightarrow \pi \pi$ matrix elements in a finite volume and the physical kaon-decay amplitudes. For technical reasons the LL formula has been derived for a finite, fixed number of pion states under the inelastic threshold and for matrix elements at zero four-momentum transfer. In the following sections a different approach to the LL formula will be discussed [6], which extends it to all elastic states under the inelastic threshold and momentum transfers different from zero.

\section{Physics in a Finite Cubic Volume}

In this section we will recall some aspects of the quantization in a cubic box, relevant in the discussion of the LL formula [7], [6]. In particular we will be interested in the structure of the zero-momentum, finite volume, energy eigenstates, $|\pi \pi, n\rangle_{V}$, which can be excited from the vacuum by a scalar operator $\sigma(x)$ i.e. for which

$V\langle\pi \pi, n|\sigma(0)| 0\rangle \neq 0$

When analyzed from the point of view of angular momentum, energy-eigenstates in a cubic box are a complicated superposition [7]

$$
|\pi \pi, n\rangle_{V}=\sum_{l=0}^{\infty} \sum_{m=-l}^{+l} \alpha_{l, m}^{(n)}|\pi \pi, n ; l, m\rangle_{V}
$$


We are interested in the structure of states which are cubically invariant and contain an s-wave component (and hence satisfy eq.(1D)).

In the framework of quantum mechanics, finitevolume quantization formulas become exact for finite-range potentials and in the presence of an angular momentum cut-off, which, in its simplest form, assumes that only s-waves interact. Under these conditions, the allowed values of the radial relative momentum $k$ of a two particle state, related to the center of mass energy $E$ as $E=2 \sqrt{m_{\pi}^{2}+k^{2}}$, are quantized as follows [7]:

1. $k$ obeys the equation (see sec.5)

$$
h(k, L) \equiv \frac{\phi(q)+\delta(k)}{\pi}=n
$$

where $n$ is a non-negative integer, $\delta(k)$ is the infinite volume s-wave phase-shift, $q \equiv \frac{k L}{2 \pi}$ and

$$
\begin{aligned}
& \tan \phi(q)=-\frac{\pi^{3 / 2} q}{Z_{00}\left(1 ; q^{2}\right)} \\
& Z_{00}\left(s ; q^{2}\right)=\frac{1}{\sqrt{4 \pi}} \sum_{\underline{n} \in Z^{3}}\left(\underline{n}^{2}-q^{2}\right)^{-s}
\end{aligned}
$$

2. $k^{2}=\underline{p}_{n}^{2}=\left(\frac{2 \pi}{L}\right)^{2} \underline{n}^{2}$ (free spectrum) if at least two $\underline{p}_{n}$ and $\underline{p}_{n}^{\prime}$ exist with $\underline{p}_{n}^{\prime}=\underline{p}_{n}^{2}$ not related by a cubic transformation. These states are non physical and their existence is a consequence of the angular momentum cut-off.

States of type 1 have a non-zero s-wave component [6], $\Psi_{E_{n}}^{V_{s-\text { wave }}}(r)$, undistorted by the presence of the boundary compared to the infinite volume s-wave function $\Psi_{E_{n}}^{\infty_{s-\text { wave }}}(r)$. Therefore inside the volume $V$,

$\Psi_{E_{n}}^{V_{s-\text { wave }}}(r)=\frac{1}{\sqrt{c\left(E_{n}\right)}} \Psi_{E_{n}}^{\infty_{\text {s-wave }}}(r)$

States of type 2 cannot be simply plane-waves of the form $e^{i \underline{p}}{ }_{n} \underline{x}$, because of the presence of the interaction. However the combination $\phi(\underline{x})=$ $e^{i \underline{p}} \underline{n}_{n}^{\cdot \underline{x}}-e^{i \underline{p}_{n}^{\prime} \cdot \underline{x}}$ is a solution of the Schroedinger equation even in the presence of the potential, because $\phi(\underline{x})$ does not contain an s-wave component and all other angular momenta are not interacting $[6]$. The spurious states $\phi(\underline{x})$ have a non-zero cubically-invariant projection, but do not project on s-wave.

In conclusion, locality and the scalar character of $\sigma(x)$ imply that

$$
\begin{aligned}
& \langle 0|\sigma(0)| \pi \pi, n ; \text { Spurious }\rangle_{V}=0, \\
& \langle 0|\sigma(0)| \pi \pi, n\rangle_{V}=\int_{V} d^{3} x S(r) \Psi_{E_{n}}^{V}(\underline{x}) \\
& \quad=\int_{V} d^{3} x S(r) \Psi_{E_{n}}^{V_{s-\text { wave }}}(r)= \\
& =\frac{1}{\sqrt{c\left(E_{n}\right)}} \int d^{3} x S(r) \Psi_{E_{n}}^{\infty_{s-\text { wave }}}(r) .
\end{aligned}
$$

where $S(r)$ is the coordinate representation of $\sigma(x)$. $S(r)$ is a function of $r$ only, so that it selects the s-wave component $\Psi_{E_{n}-\text { wave }}^{V_{s}}(r)$ in the angular momentum expansion, eq.(2). $S(r)$ is also localized inside $V$, thus justifying the last step in eq.(8). With the definition

$$
\begin{aligned}
& \sigma\left(E_{n}\right) \equiv\left\langle 0|\sigma(0)| \pi \pi, E_{n}\right\rangle= \\
& =\int d^{3} x S(r) \Psi_{E_{n}}^{\infty_{s-\text { wave }}}(r)
\end{aligned}
$$

we therefore have

$$
\left|\sigma\left(E_{n}\right)\right|^{2}=c\left(E_{n}\right)\left|\langle 0|\sigma(0)| \pi \pi, n\rangle_{V}\right|^{2} .
$$

After these preliminaries we are ready to discuss the LL proposal, which consists in tuning the volume $V$ so that one of the first seven excited twopion state is degenerate in energy with the kaon state (for $n=1, L \approx 5 \div 6 \mathrm{Fm}$ ) and then using the LL relation which connects finite and infinite volume matrix elements

$$
\begin{aligned}
& \left|\left\langle\pi \pi, E=m_{K}\left|\mathcal{H}_{W}(0)\right| K\right\rangle\right|^{2}= \\
& =\left.\left.V^{2}\right|_{V}\left\langle\pi \pi, E\left|\mathcal{H}_{W}(0)\right| K\right\rangle_{V}\right|^{2}\left(\frac{m_{K}}{k}\right)^{3} \times \\
& \times 8 \pi\left[q \phi^{\prime}(q)+k \delta^{\prime}(k)\right] .
\end{aligned}
$$

In eq.(11) $|\pi \pi, E\rangle_{V}$ denotes a finite volume two pion state with zero total momentum and "angular momentum", whose energy $E$ is to be chosen 
among the solutions of eq.(3), while $|K\rangle_{V}$ denotes a finite volume kaon state with zero momentum. Both states are normalized to 1 . The LL formula has been derived [5] for a large enough volume, $n=1 \div 7$ and $\Delta E=\Delta \underline{P}=0$.

Eq.(10) shows that $c\left(E_{n}\right)$, defined in eq.(6), is precisely the LL proportionality factor.

\section{The Nature of the LL Relation}

In order to relate the states at finite and infinite volume we consider the correlator

$$
\begin{aligned}
& \int_{V} d^{3} x\langle\sigma(\underline{x}, t) \sigma(0)\rangle_{V} \underset{V \rightarrow \infty}{\longrightarrow} \\
& \frac{(2 \pi)^{3}}{2(2 \pi)^{6}} \int \frac{d \underline{p}_{1}}{2 \omega_{1}} \frac{d \underline{p}_{2}}{2 \omega_{2}} \delta\left(\underline{p}_{1}+\underline{p}_{2}\right) e^{-\left(\omega_{1}+\omega_{2}\right) t} \times \\
& \times\left|\left\langle 0|\sigma(0)| \underline{p}_{1}, \underline{p}_{2}\right\rangle\right|^{2}= \\
= & \frac{1}{2(2 \pi)^{3}} \int d E e^{-E t}|\langle 0|\sigma(0)| \pi \pi, E\rangle|^{2} \times \\
& \times \int \frac{d \underline{p}_{1}}{2 \omega_{1}} \frac{d \underline{p}_{2}}{2 \omega_{2}} \delta\left(\underline{p}_{1}+\underline{p}_{2}\right) \delta\left(E-\omega_{1}-\omega_{2}\right)= \\
= & \frac{\pi}{2(2 \pi)^{3}} \int \frac{d E}{E} e^{-E t}|\langle 0|\sigma(0)| \pi \pi, E\rangle|^{2} k(E) .
\end{aligned}
$$

On the other hand we could proceed differently

$$
\begin{gathered}
\int_{V} d^{3} x\langle\sigma(\underline{x}, t) \sigma(0)\rangle= \\
V \sum_{n}\left|\langle 0|\sigma(0)| \pi \pi, n\rangle_{V}\right|^{2} e^{-E_{n} t} \underset{V \rightarrow \infty}{\longrightarrow} \\
\underset{V \rightarrow \infty}{\longrightarrow} V \int_{0}^{\infty} d E \rho_{V}(E)\left|\langle 0|\sigma(0)| \pi \pi, E\rangle_{V}\right|^{2} e^{-E t},
\end{gathered}
$$

where only states containing an s-wave component contribute. In eq.(13) $\rho_{V}(E)$ denotes a function to be determined, which provides the correspondence between finite volume sums and infinite volume integrals. In many cases, for example in one dimension, $\rho_{V}(E)$ can be identified as the density of states at energy $E$. In section 14 we show that, also in the presence of cubic boundary conditions, $\rho_{V}(E)$ is given by

$\rho_{V}(E)=\frac{d n}{d E}=\frac{q \phi^{\prime}(q)+k \delta^{\prime}(k)}{4 \pi k^{2}} E$, with exponential precision in the volume. The expression in eq. (14) is the one one would heuristically derive from a naïve interpretation of $\rho_{V}(E)$ as the density of states, as seen from eq. (3).

Comparing eqs.(12) and (13) we get the correspondence

$|\pi \pi, E\rangle \Leftrightarrow 4 \pi \sqrt{\frac{V E \rho_{V}(E)}{k(E)}}|\pi \pi, E\rangle_{V}$.

In a similar way one gets

$$
|K, \underline{p}=0\rangle \Leftrightarrow \sqrt{2 m V}|K, \underline{p}=0\rangle_{V},
$$

from which it is easy to recover the LL relation, eq.(11), without any restriction on the fourmomentum transfer.

Although the present approach appears superficially to be equivalent to the one of ref. [5], there is an important difference in the two derivations. The result of ref. [5] was obtained at a fixed value of $n$ and therefore at a fixed volume $V$, tuned so that $m_{K}=E_{n}$, with $n<8$. We, on the other hand, derived the same result at fixed energy $E$, for asymptotically large volumes $V$. This implies that, as $V \rightarrow \infty$, we must simultaneously allow $n \rightarrow \infty$. A question which arises naturally at this point is what is the relation between the two approaches? The answer requires a more detailed discussion, developed in the following section, where it will be shown that the constraints of locality allow us to establish eq. (14) with exponential accuracy for elastic states under the inelastic threshold.

\section{Summation Theorems, Locality and the LL Formula}

Locality has already been an important ingredient in establishing eq.(10). In this section we will discuss another important and more subtle rôle of locality. Our approach to the LL formula, outlined in the previous section, is based on the identification

$$
\begin{aligned}
& \sum_{n}\left|\langle 0|\sigma(0)| \pi \pi, n\rangle_{V}\right|^{2} e^{-E_{n} t} \approx \\
& \approx \int_{\bar{E}}^{\infty} d E|\sigma(E)|^{2} e^{-E t}
\end{aligned}
$$


of finite and infinite volume correlators. We are therefore naturally led to the question of how well a sum may approximate an integral. We will show that the key ingredient to answer this question is again locality.

We start with the example of a simple summation theorem. Take any $\tilde{f}(\underline{x})$ and $\tilde{g}(\underline{x})$ exponentially decreasing at large $|\underline{x}|$ and compute their Fourier transforms

$f(\underline{p})=\int_{\substack{V \\ \infty}} d \underline{x} \tilde{f}(\underline{x}) e^{i \underline{p}} \underline{x}, g(\underline{p})=\int_{\substack{V \\ \infty}} d \underline{x} \tilde{g}(\underline{x}) e^{i \underline{p} \cdot \underline{x}}$.

In eq. (18) the integrals can be taken on a finite volume $V$ or over all the three-dimensional space, the difference being exponentially small in $V$. If we define

$\underline{p}_{n} \equiv \frac{2 \pi}{L} \underline{n}$

we have

$$
\begin{aligned}
& \int_{\infty} \tilde{f}(\underline{x}) \tilde{g}^{*}(\underline{x}) d \underline{x}=\frac{1}{(2 \pi)^{3}} \int f(\underline{p}) g^{*}(\underline{p}) d \underline{p}= \\
& =\int_{V} \tilde{f}(\underline{x}) \tilde{g}^{*}(\underline{x}) d \underline{x}=\frac{1}{V} \sum_{\left\{\underline{p}_{n}\right\}} f\left(\underline{p}_{n}\right) g^{*}\left(\underline{p}_{n}\right)
\end{aligned}
$$

and therefore

$$
\frac{1}{(2 \pi)^{3}} \int f(\underline{p}) g^{*}(\underline{p}) d \underline{p}=\frac{1}{V} \sum_{\left\{\underline{p}_{n}\right\}} f\left(\underline{p}_{n}\right) g^{*}\left(\underline{p}_{n}\right),
$$

again with exponential accuracy in $V$. If the support of $\tilde{f}(\underline{x})$ and $\tilde{g}(\underline{x})$ is entirely contained inside $V$, eq.(21) becomes exact.

Relations similar in nature to eq.(21) can be obtained in quantum mechanics and field theory. In fact, since $\sigma(x)$ is a local operator, $\sigma(0)|0\rangle$ is a localized state which does not differ much from the vacuum state away from 0 . This is a consequence of clustering, which guarantees that, in the absence of massless particles, the probability of finding particles at a distance $r$ away from the origin in the state $\sigma(0)|0\rangle$ decreases exponentially like $e^{-2 m_{\pi} r}$. As a consequence, if $V$ is greater that the localization volume, we can write, at $t=0$,

$$
\begin{gathered}
\int_{V} d^{3} x\langle\sigma(\underline{x}, 0) \sigma(0)\rangle_{V}=V \sum_{n}\left|\langle 0|\sigma(0)| \pi \pi, n\rangle_{V}\right|^{2} \\
=\int_{\bar{E}}^{\infty} d E|\sigma(E)|^{2}=\int d^{3} x\langle\sigma(\underline{x}, 0) \sigma(0)\rangle \quad(22)
\end{gathered}
$$

with exponential accuracy in $V$. The second and third lines of eqs.(22) were obtained by inserting the complete set of energy eigenstates on a finite and infinite volume respectively. The result is the same apart from the exponentially small perturbation at the boundary. In other words, we can introduce states

$$
|\pi \pi, \underline{x}\rangle_{V}=\sqrt{V} \sum_{n}|\pi \pi, n\rangle_{V} \Psi_{E_{n}}^{V}(\underline{x})
$$

where $\Psi_{E_{n}}^{V}(\underline{x})$ are the finite volume center of mass wave functions. $|\pi \pi, \underline{x}\rangle_{V}$ represents a state with $\underline{P}=0$, in which the two pions are localized a distance $\underline{x}$ apart. If the distance $\underline{x}$ is inside $V$, the same state is exponentially well represented by

$$
|\pi \pi, \underline{x}\rangle=(2 \pi)^{3 / 2} \int_{\bar{E}}^{\infty} d E|\pi \pi, E\rangle \Psi_{E}^{\infty *}(\underline{x})
$$

where $\Psi_{E}^{\infty}(\underline{x})$ are the infinite-volume center of mass wave functions.

By virtue of the cluster property mentioned above, we know that

$F(\underline{x}) \equiv\langle\pi \pi, \underline{x}|\sigma(0)| 0\rangle$

is a localized function of $\underline{x}$, so that

$F(\underline{x}) \approx{ }_{V}\langle\pi \pi, \underline{x}|\sigma(0)| 0\rangle$

with exponential precision and

$$
\begin{aligned}
& \int_{V} d^{3} x\langle\sigma(\underline{x}, 0) \sigma(0)\rangle_{V}= \\
& =V \sum_{n}\left|\langle 0|\sigma(0)| \pi \pi, n\rangle_{V}\right|^{2}= \\
& =\int\left|\langle 0|\sigma(0)| \pi \pi, \underline{x}\rangle_{V}\right|^{2} d \underline{x}= \\
& =\int|\langle 0|\sigma(0)| \pi \pi, \underline{x}\rangle|^{2} d \underline{x}= \\
& =\int_{\bar{E}}^{\infty} d E|\sigma(E)|^{2}=\int d^{3} x\langle\sigma(\underline{x}, 0) \sigma(0)\rangle
\end{aligned}
$$

confirming eq.(22). Eqs.(22) and (5) express the important property that the matrix elements of a local operator are smooth in energy, in the sense that the sum over discrete energy levels reproduces, with exponential accuracy, the corresponding integral

$$
\int_{\bar{E}}^{\infty} d E|\sigma(E)|^{2}=V \sum_{n} \frac{\left|\sigma\left(E_{n}\right)\right|^{2}}{c\left(E_{n}\right)} .
$$


Introduction of time dependence does not substantially change this result. We have

$$
\begin{gathered}
\int_{V} d^{3} x\langle\sigma(\underline{x}, t) \sigma(0)\rangle_{V}= \\
=V \sum_{n}\left|\langle 0|\sigma(0)| \pi \pi, n\rangle_{V}\right|^{2} e^{-E_{n} t}= \\
=\int\langle 0|\sigma(0)| \pi \pi, \underline{x}\rangle e^{-\tilde{H} t}\langle\pi \pi, \underline{x}|\sigma(0)| 0\rangle d \underline{x}= \\
=\int F^{*}(\underline{x})\left(e^{-\tilde{H} t} F\right)(\underline{x}) d \underline{x}=\int F^{*}(\underline{x}) F(\underline{x}, t) d \underline{x},
\end{gathered}
$$

where the two-body hamiltonian $\tilde{H}$ is related to the second quantized hamiltonian, $H$, through

$$
\begin{gathered}
e^{-H t}|\pi \pi, \underline{x}\rangle=\sqrt{V} \sum_{n}|\pi \pi, n\rangle_{V} \Psi_{E_{n}}^{*}(\underline{x}) e^{-E_{n} t}= \\
=\sqrt{V} \sum_{n}|\pi \pi, n\rangle_{V} e^{-\tilde{H} t} \Psi_{E_{n}}^{*}(\underline{x}) .
\end{gathered}
$$

$F(\underline{x}, t)$ as defined in eq.(29) has a time evolution characteristic of a diffusion process with hamiltonian $\tilde{H}$. Therefore both $F(\underline{x}, t)$ and the correlator $\int_{V} d^{3} x\langle\sigma(\underline{x}, t) \sigma(0)\rangle_{V}$ are insensitive to the presence of the boundaries, up to exponentially small terms, for a long time $\left(t \approx m_{\pi} L\right)$, and we have

$$
\int_{\bar{E}}^{\infty} d E|\sigma(E)|^{2} e^{-E t}=V \sum_{n} \frac{\left|\sigma\left(E_{n}\right)\right|^{2}}{c\left(E_{n}\right)} e^{-E_{n} t}
$$

with exponential precision in $V$.

The basic tool for relating integrals to sums is provided by the Poisson identity

$$
\sum_{n=-\infty}^{+\infty} \delta(n-x)=\sum_{l=-\infty}^{+\infty} e^{2 \pi i l x} .
$$

Eq.(32), together with the substitution $x \rightarrow$ $h(E, L)$, where $h(E, L)$ is defined in eq.(3), gives

$$
\begin{aligned}
& \int_{E}^{\infty} d E|\sigma(E)|^{2} e^{-E t}= \\
& =\sum_{n} \frac{\left|\sigma\left(E_{n}\right)\right|^{2} e^{-E_{n} t}}{\left.\frac{\partial h(E, L)}{\partial E}\right|_{E_{n}}}-\mathcal{Q}(L, t),
\end{aligned}
$$

where

$$
\mathcal{Q}(L, t) \equiv \sum_{l \neq 0} \int_{\bar{E}}^{\infty} d E|\sigma(E)|^{2} e^{-E t} e^{2 i l \delta(k)} e^{2 i l \phi(q)}
$$

Consider the large $L$ behavior of $\mathcal{Q}(L, t)$. The techniques of asymptotic analysis [B], together with the fact that $\phi(q) \rightarrow \infty$ when $L \rightarrow \infty$ at fixed $k$, suggest that the large $L$ behavior of each term of the sum over $l$ in eq.(34) is dominated by the critical points of $\phi(q)$, i.e. the points at which $\phi(q)$ has a vanishing derivative or the points where $\phi(q)$ and $\sigma(E)$ are not continuous or differentiable. $q=0$ is a critical point of $\phi(q)$ as follows from

$\phi(q) \underset{q \approx 0}{\approx} 2 \pi^{2} q^{3}$

so that, in absence of further critical points we expect that

$$
Q(L, t) \underset{L \rightarrow \infty}{\longrightarrow} 0
$$

as a power of $\frac{1}{L}$ depending on the threshold behavior of $\sigma(E)$ which, for local observables, may be make arbitrarily small [6].

A comparison between eqs. (31) and (33) then leads to the identification

$$
\left.c\left(E_{n}\right) \Leftrightarrow V \frac{\partial h(E, L)}{\partial E}\right|_{E_{n}}
$$

and gives the LL relation with exponential accuracy, for energies between the elastic and inelastic thresholds.

Although a rigorous mathematical proof that $q=0$ is the only critical point of $\phi(q)$ in the complex $q$-plane is lacking, we can provide a simple, indirect argument that this is likely to be the case. For this we start applying eq.(21) to the case of two functions $f(p)$ and $g(p)$ which only depend on $p \equiv|\underline{p}|$

$$
\begin{aligned}
\frac{1}{2 \pi^{2}} & \int_{0}^{\infty} f(p) g^{*}(p) p^{2} d p=\frac{1}{V} \sum_{\left\{\underline{p}_{n}\right\}} f\left(p_{n}\right) g^{*}\left(p_{n}\right)= \\
& =\frac{1}{V} \sum_{\left\{p_{n}\right\}} \nu_{n} f\left(p_{n}\right) g^{*}\left(p_{n}\right),
\end{aligned}
$$

where $\nu_{n}$ is the number of integer vectors with given $|\underline{n}|$. The last sum in eq.(38) is performed over the distinct values of $p_{n}$. On the other hand the $p_{n}$ 's are the solutions of

$\phi(q)=n \pi$ 
because, from eq.(14) we have that

$\tan \phi(q)=0 \Rightarrow q=|\underline{n}|$

where $\underline{n}$ is any vector with integer components. We can now use the Poisson Identity eq.(32) with $x \rightarrow \phi(q) / \pi$ which, together with

$\phi^{\prime}(|\underline{n}|) \mid=\frac{4 \pi^{2}}{\nu_{n}} \underline{n}^{2}$

and after multiplication by $p^{2} / 2 \pi^{2} f(p) g^{*}(p)$ and integration over $p$, gives

$$
\begin{aligned}
& \frac{1}{3 V} f(0) g^{*}(0)+\frac{1}{V} \sum_{\left\{p_{n} \neq 0\right\}} \nu_{n} f\left(p_{n}\right) g^{*}\left(p_{n}\right)= \\
& =-\frac{2}{3 V} f(0) g^{*}(0)+\frac{1}{V} \sum_{\left\{p_{n}\right\}} \nu_{n} f\left(p_{n}\right) g^{*}\left(p_{n}\right)= \\
& =\frac{1}{(2 \pi)^{3}} \int f(p) g^{*}(p) d \underline{p}+ \\
& \quad+\sum_{l \neq 0} \frac{1}{2 \pi^{2}} \int_{0}^{\infty} p^{2} f(p) g^{*}(p) e^{2 i l \phi\left(\frac{L p}{2 \pi}\right)} d p .
\end{aligned}
$$

Eq.(42), together with eq.(38) shows that

$$
\sum_{l \neq 0} \frac{1}{2 \pi^{2}} \int_{0}^{\infty} p^{2} f(p) g^{*}(p) e^{2 i l \phi\left(\frac{L p}{2 \pi}\right)} d p
$$

is indeed exponentially small with power corrections concentrated at threshold. This is in complete agreement with the heuristic analysis presented after eq.(34). This argument strongly suggests that the only critical point of $\phi(q)$ is at $q=0$. In fact if $\phi(q)$ possessed other critical points in the complex $q$-plane, these would show up in eq. (43), through a large $L$ behavior different from the one just found.

\section{Finite Volume Quantization in Quan- tum Field Theory and the Effects of In- elasticity}

The approach presented in previous sections is based on the property that local correlators may be expressed, with exponential accuracy, both as a sum or as an integral over intermediate states. This may appear to be a great difference of our approach compared to that of ref. [5]. We will argue, however, that the validity of eq.(3) also requires the volume to be sufficiently large for the
Fourier series to be equal to the infinite-volume energy integrals, up to exponential corrections. Since the derivation of the LL relation in ref. [5] relies on this quantization formula, we conclude that the conditions on the volume for the applicability of this relation are equivalent in the two approaches.

The validity of eq.(3) in Relativistic Quantum Field Theory (RQFT) has been discussed in ref. [7]. In this section we describe a different approach which helps to clarify the size of the corrections to eq.(3) due to the presence of an inelastic threshold, $E_{\text {in }}$ [6].

The concept of a wave function in RQFT is an approximate one. The object closest to a wave function is the Bethe-Salpeter (BS) wave function. In infinite-volume, for an incoming state with total momentum zero, the $t=0 \mathrm{BS}$ wave function is defined as

$$
\begin{aligned}
& \Phi_{\underline{k}}(\underline{x})=\langle 0|\phi(\underline{x}, 0) \phi(0)| \underline{k},-\underline{k}\rangle_{i n}= \\
& =\sum_{n}\langle 0|\phi(\underline{x}, 0)| n\rangle\langle n|\phi(0)| \underline{k},-\underline{k}\rangle_{i n},
\end{aligned}
$$

where $\phi(x)$ is an appropriately normalized pion field. In eq.(44) we can separate the contribution of single pion states, $\Psi_{\underline{k}}(\underline{x})$ and that of multipion states, $I_{\underline{k}}(\underline{x})$, as

$\Phi_{\underline{k}}(\underline{x})=\Psi_{\underline{k}}(\underline{x})+I_{\underline{k}}(\underline{x})$

where

$\Psi_{\underline{k}}(\underline{x})=\int \frac{d^{3} p}{(2 \pi)^{3} 2 E_{p}}\langle\underline{p}|\phi(0)| \underline{k},-\underline{k}\rangle_{i n} e^{i \underline{p} \cdot \underline{x}}$

$I_{\underline{k}}(\underline{x})=\sum_{\{3 \pi\}}\langle 0|\phi(0)| 3 \pi\rangle\langle 3 \pi|\phi(0)| \underline{k},-\underline{k}\rangle_{i n} e^{i \underline{p} \underline{p}_{3 \pi} \underline{x}}$

$$
+\cdots
$$

where the second sum runs over three pion states and the ellipses represent the contribution from states with a higher number of particles. As will be shown later, $I_{\underline{k}}(\underline{x})$ vanishes exponentially with $\underline{x}$ and will be neglected for the moment. We can further separate, in $\Psi_{\underline{k}}(\underline{x})$, the connected part

$$
\begin{aligned}
& \Psi_{\underline{k}}(\underline{x})=e^{i \underline{k} \cdot \underline{x}}+ \\
& +\int \frac{d^{3} p}{(2 \pi)^{3} 2 E_{p}}\langle\underline{p}|\phi(0)| \underline{k},-\underline{k}\rangle_{i n}^{c o n n} e^{i \underline{p} \underline{x}}
\end{aligned}
$$


and parametrize $\langle\underline{p}|\phi(0)| \underline{k},-\underline{k}\rangle_{i n}^{\text {conn }}$ as

$$
\langle\underline{p}|\phi(0)| \underline{k},-\underline{k}\rangle_{i n}^{c o n n}=\frac{1}{4 E_{k}} \frac{\mathcal{M}}{E_{p}-E_{k}-i \varepsilon} .
$$

The off-shell scattering amplitude $\mathcal{M}$ becomes the physical one 2., $\mathcal{M}(\underline{k} \rightarrow \underline{p})$, when $p=k$

$\mathcal{M}(\underline{k} \rightarrow \underline{p})=\frac{4 \pi}{i} \frac{2 E_{k}}{k}\left(e^{i 2 \delta(k)}-1\right)$.

We therefore have

$$
\begin{aligned}
& \Psi_{\underline{k}}(\underline{x})=e^{i \underline{k} \cdot \underline{x}}+ \\
& +\int \frac{d^{3} p}{(2 \pi)^{3} 2 E_{p}} \frac{\mathcal{M}}{4 E_{k}\left(E_{p}-E_{k}-i \varepsilon\right)} e^{i \underline{p} \cdot \underline{x} .}
\end{aligned}
$$

The projection of $\Psi_{\underline{k}}(\underline{x})$ over the s-wave is

$$
\begin{aligned}
& \left.\Psi_{\underline{k}}\right|_{s-\text { wave }}(r)=\frac{\left(e^{i 2 \delta(k)}+1\right)}{2} \frac{\sin k r}{k r}+ \\
& +\mathcal{P} \int \frac{d^{3} p}{(2 \pi)^{3} 2 E_{p}} \frac{\left(E_{p}+E_{k}\right) \mathcal{M}}{4 E_{k}\left(p^{2}-k^{2}\right)} \frac{\sin p r}{p r},
\end{aligned}
$$

where we used the identity

$$
\frac{1}{x-i \varepsilon}=i \pi \delta(x)+\mathcal{P} \frac{1}{x} \text {. }
$$

From eq.(52) we obtain the large $r$ behaviour of $\left.\Psi_{\underline{k}}\right|_{s-\text { wave }}(r)$

$\left.\Psi_{\underline{k}}\right|_{s-\text { wave }}(r)=\frac{1}{k r}\left(\sin k r+\frac{1}{2 i}\left(e^{i 2 \delta(k)}-1\right) e^{i k r}\right)$

As discussed in section 2, the relative momenta $k$ allowed in a box are determined by the condition that the s-wave projection of the finite volume BS wave function, $\left.\Phi_{k}^{V}\right|_{s \text {-wave }}(r)$, is not deformed by the presence of the boundary. $\Phi_{\underline{k}}^{V}(\underline{x})$ is given by an expression similar to eq.(44), where the sum over intermediate states runs over finite volume energy eigenstates, so that, neglecting for the moment the contribution from multipion states, we have

$$
\begin{aligned}
& \left.\Psi_{\underline{k}}^{V}\right|_{s-\text { wave }}(r) \equiv \\
& \equiv \frac{1}{V} \sum_{\left\{\underline{p}_{n}\right\}} \frac{1}{2 E_{p_{n}}} \frac{\left(E_{p_{n}}+E_{k}\right) \mathcal{M}}{4 E_{k}\left(p_{n}^{2}-k^{2}\right)} \frac{\sin p_{n} r}{p_{n} r},
\end{aligned}
$$

\footnotetext{
${ }^{2}$ As before we keep the interaction only for the s-wave.
}

where the sum is over single particle momenta given by eq.(19). The quantization condition requires therefore the identity of eqs.(52) and (55). We stress that in the finite volume expression, eq.(55), disconnected terms and $i \varepsilon$ 's are absent because the eigen-momenta $k$ do not coincide with any of the $p_{n}$. These terms will appear as $V$ grows, as shown in a moment.

In appendix $\mathrm{C}$ of ref. [6] we prove the summation formula

$$
\begin{aligned}
& \frac{1}{V} \sum_{\left\{\underline{p}_{n}\right\}} \frac{f\left(p_{n}^{2}\right)}{k^{2}-p_{n}^{2}}= \\
& =\frac{1}{(2 \pi)^{3}} \mathcal{P} \int_{-\infty}^{\infty} d^{3} p \frac{f\left(p^{2}\right)}{k^{2}-p^{2}}+c f\left(k^{2}\right)
\end{aligned}
$$

where $\quad c=-\frac{Z_{00}\left(1, q^{2}\right)}{2 \pi^{\frac{3}{2}} L}$.

Eq.(56) is valid up to exponentially small corrections, provided the Fourier transform of $f\left(p^{2}\right)$ vanishes exponentially with $r$ and, together with eqs.(55) and (52), gives at once the Lüscher quantization condition, eq.(3), with exponential precision in $V$. The size of the corrections are determined by the precision with which the sum in eq.(55) is able to reproduce the corresponding integral, a condition analogous to the one which was underlying our derivation of the LL formula. It appears therefore that the two formulas have the same conditions of applicability. In particular, a possible source of concern is the (practically relevant) situation in which the quantization volume allows only very few (perhaps two or three) elastic states under the inelastic threshold. In this case, even though the finite-volume effects are exponentially small, one is working in a fixed volume and in order to establish that the corrections are indeed negligible one needs an estimate of such effects. These corrections are small if $\left.\Psi_{\underline{k}}(r)\right|_{\text {s-wave }}$ is well approximated by its asymptotic expression, eq.(54), at $r \approx L$. There are essentially two types of exponentially small corrections. The first type is analogous to the corrections which would be present, even in quantum mechanics in the presence of an exponentially decreasing potential, rather than a finite range one. The second type of corrections, characteristic of RQFT, 
are due to the existence of inelasticity and are responsible for the failure of eq.(3) above $E_{i n}$. They come from the term $I_{\underline{k}}(\underline{x})$ in eq. (45) and a corresponding crossed contribution in $\Psi_{\underline{k}}(\underline{\underline{x}})$. The matrix element $\langle 3 \pi|\phi(0)| \underline{k},-\underline{k}\rangle_{\text {in }}$ in eq.(47) has a singularity when the virtual pion described by $\phi$ reaches the mass-shell. This singularity is complex for $2 E_{k}<E_{i n}$, implying an exponential vanishing of $I_{\underline{k}}(\underline{x})$. For values of $2 E_{k}$ closer to $E_{i n}$ the singularity is closer to the real axis, and the range of the exponential increases. Therefore the quantization condition is, in general, affected by the presence of inelasticity. In the case of pions, however, there are indications that inelasticity is negligible up to around $1 \mathrm{Gev}[9]$. This shifts the singularity further from the real axis, making the finite-volume effects less important and allowing the LL strategy to be applicable to $K \rightarrow \pi \pi$ decays without sizeable corrections.

\section{Conclusions}

We have reviewed finite-volume effects in $K \rightarrow$ $\pi \pi$ decays, examining the conditions under which the LL formula is valid. We were able to remove the restrictions of zero momentum transfer and $n<8$, present in the original LL derivation, allowing the infinite volume limit to be taken. We have also presented an alternative simple derivation of the Lüscher quantization condition in Quantum Field Theory, which allows us to examine the general consequences of inelasticity on the quantization formula. For the particular case of $K \rightarrow \pi \pi$ decays we conclude that these corrections are not important.

\section{Acknowledgements}

M. Testa thanks the organizers of LHP2001 for the hospitality in Cairns. D. Lin, C. Sachrajda and M. Testa thank the Institute for Nuclear Theory at the University of Washington for its hospitality and the Department of Energy for partial support during the completion of this work. This work was supported by European Union grant HTRN-CT-2000-00145.
1. M. Bochicchio et al., Nucl. Phys. B262 (1988) B262;

L. Maiani, G. Martinelli, G.C. Rossi and M. Testa, Phys. Lett. 176B (1986) 445 and Nucl. Phys. B289 (1987) 505.

2. C. Bernard, T. Draper, G. Hockney and A. Soni, Nucl. Phys. B.Proc.Suppl. 4 (1988) 483.

3. C. Dawson et al., Nucl. Phys. B514 (1998) 313.

4. L. Maiani and M. Testa, Phys. Lett. B245 (1990) 585.

5. L. Lellouch and M. Lüscher, Commun. Math. Phys. 219 (2001) 31.

6. C.-J.D. Lin, G. Martinelli, C.T. Sachrajda, M. Testa, hep-lat/0104006, Nucl. Phys.B, to appear.

7. M. Lüscher, Commun. Math. Phys. 104 (1986) 177; Commun. Math. Phys. 105 (1986) 153; Nucl. Phys. B354 (1991) 531; Nucl. Phys. B364 (1991) 237.

8. N. Bleistein, R.A. Handelsman Asymptotic expansions of integrals (Dover Editions, 1986).

9. See for example D. Morgan and M.R. Pennington, in The second Daphne Physics Handbook, edited by L Maiani, G. Pancheri and N. Paver, INFN-LNF.

\section{REFERENCES}

